INPLASY

PROTOCOL

To cite: Tang et al. The effectiveness of acupuncture and moxibustion for

rheumatoid arthritis: a protocol

of an overview of systematic

reviews and meta-analysis.

Inplasy protocol 202080031.

doi:

10.37766/inplasy2020.8.0031

Received: 08 August 2020

Published: 08 August 2020

Corresponding author:

Xiong Jun

xiongjun196071@163.com

Author Affiliation:

Jiangxi University of

Traditional Chinese Medicine

Support: 1050Project:

5141900101

Review Stage at time of this submission: The review has not yet started.

Conflicts of interest:

The authors declare no conflicts of interest.

\section{The effectiveness of acupuncture and moxibustion for rheumatoid arthritis: a protocol of an overview of systematic reviews and meta-analysis}

Tang, $\mathrm{GH}^{1} ;$ Xiong, $\mathrm{J}^{2}$.

Review question / Objective: This overview in an effort to summarize the available evidence from current systematic reviews for the efficacy of acupuncture therapy for rheumatoid arthritis.

Condition being studied: Rheumatoid arthritis is a common disease with an annual incidence of 25 per 10000 of population. RA is characterized by polyarticular inflammation and pannus formation, leading to joint destruction and severe disability. Western medicine and psychotherapy are not universally effective in treating this disease, Acupuncture and moxibustionis reported to be efficacious and widely used for the treatment of RA. This overview aims to summarize the available evidence from current systematic reviews for the efficacy of acupuncture and moxibustion therapy for rheumatoid arthritis.

INPLASY registration number: This protocol was registered with the International Platform of Registered Systematic Review and Meta-Analysis Protocols (INPLASY) on 08 August 2020 and was last updated on 08 August 2020 (registration number INPLASY202080031).

\section{INTRODUCTION}

Review question / Objective: This overview in an effort to summarize the available evidence from current systematic reviews for the efficacy of acupuncture therapy for rheumatoid arthritis.
Condition being studied: Rheumatoid arthritis is a common disease with an annual incidence of 25 per 10000 of population.RA is characterized by polyarticular inflammation and pannus formation, leading to joint destruction and 
severe disability. Western medicine and psychotherapy are not universally effective in treating this disease, Acupuncture and moxibustionis reported to be efficacious and widely used for the treatment of RA. This overview aims to summarize the available evidence from current systematic reviews for the efficacy of acupuncture and moxibustion therapy for rheumatoid arthritis.

\section{METHODS}

Search strategy: Terms and electronic databases included in the review.

Participant or population: Patients with rheumatoid arthritis.

Intervention: Needle acupuncture, electroacupuncture, auricular acupuncture, moxibustion, acupressure, point injection, or any combination of the above.

Comparator: Western medicine, placebo, sham acupuncture, no treatment, or any combination of these.

Study designs to be included: All systematic reviews and meta-analysis on the use of acupuncture and moxibustion for rheumatoid arthritis.

Eligibility criteria: Published systematic reviews which were reported in Chinese or English, and meet the "PICOS", will be considered for inclusion in this overview.

Information sources: Pubmed, Embase, Cochrane Library, Chinese Biomedical Literatures Database(CBM), China National Knowledge Infrastructure ( CNKI), Wan Fang Database ( WF), Chinese Scientific Journal Database (VIP).

Main outcome(s): The effective rate, The House- Brackmann(H-B) score, cure rate.

Data management: (1)We will use NoteExpress3.2.0.7535 and Excel software 11.1.0.9912 to extract data. The content will be saved in electronic form. (2) Different review authors will independently screen the titles and abstracts of records obtained by searching the electronic databases to determine potential eligibility. Full texts screening and data extraction will be conducted afterwards independently. Any disagreement regarding study selection will be resolved through discussion or arbitrated by the third author if necessary. In this step, we will use NoteExpress. (3)The research team designed structured data extraction tables, including: the first author, nationality, publication year, patients' basic information, sample size, intervention measures of test group, intervention measures of controlled group, qualitative evaluation method, target outcome (including primary outcome measures and secondary outcome measures), etc. Different review authors will independently extract data.Any disagreement regarding data extraction will be resolved through discussion or arbitrated by the third author if necessary. In this step, we will use Excel.

Quality assessment / Risk of bias analysis: Assessment of Multiple Systematic Reviews 2 (AMSTAR-2) measurement tool, Preferred Reporting Items for Systematic Reviews and Meta-Analyses (PRISMA), Grading of Recommendations Assessment, Development and Evaluation(GRADE) approach.

Strategy of data synthesis: We will provide a narrative description of the findings of the included systematic reviews (SRs). Tables will be produced to detail the included studies and their outcomes. In addition, we will synthesis these reviews and provide pooled treatment effects for all SRs which include the following outcomes: The effective rate, the HouseBrackmann( $\mathrm{H}-\mathrm{B}$ ) score, cure rate. If necessary, this study will use RevMan5.4 software for data integration and analysis. The measurement data will use the mean difference (MD) as the effect indicator, and the count data will use the odds ratio (OR)as the effect index. Each effect indicator will be given as a point estimate with $95 \%$ confidence interval.The heterogeneity and size of each study result will be judged using statistical methods. For studies with no statistical 
heterogeneity, the analysis will be performed using a fixed-effect model, whereas a randomized effects model will be applied if for studies with significant statistical heterogeneity.

Subgroup analysis: If the necessary data are available, subgroup analysis will be carried out according to different factors as follows: 1. Control interventions (eg, sham/ placebo moxibustion, no treatment, other TCM treatment or non-TCM treatment). 2. Type of acupuncture and moxibustion (eg, needle acupuncture, electro-acupuncture, auricular acupuncture, heat-sensitive moxibustion, thunder fire miraculous moxa roll, warm needling moxibustion suspended moxibustion or mild moxibustion).

Sensibility analysis: To assess the influence of each individual study, leave-one-out sensitivity analysis was performed iteratively by removing one study at a time to confirm that the findings were not influenced by any single study. No sensitivity analysis required in overview.

Country(ies) involved: China.

Keywords: Rheumatoid arthritis ; acupuncture; moxibustion; AMSTAR-2;

PRISMA; GRADE; overview.

Contributions of each author:

Author 1 - Genhua Tang - the author draftd the manuscript.

Author 2 - Jun Xiong - revise this protocol. 